\title{
Liquid Democracy: An Algorithmic Perspective
}

\author{
Anson Kahng \\ Computer Science Department \\ Carnegie Mellon University \\ Simon Mackenzie \\ University of New South Wales
}

Ariel D. Procaccia

School of Engineering and Applied Sciences

Harvard University
AKAHNG@CS.CMU.EDU

SIMON.WILLIAM.MACKENZIE@GMAIL.COM

ARIELPRO@SEAS.HARVARD.EDU

\begin{abstract}
We study liquid democracy, a collective decision making paradigm that allows voters to transitively delegate their votes, through an algorithmic lens. In our model, there are two alternatives, one correct and one incorrect, and we are interested in the probability that the majority opinion is correct. Our main question is whether there exist delegation mechanisms that are guaranteed to outperform direct voting, in the sense of being always at least as likely, and sometimes more likely, to make a correct decision. Even though we assume that voters can only delegate their votes to better-informed voters, we show that local delegation mechanisms, which only take the local neighborhood of each voter as input (and, arguably, capture the spirit of liquid democracy), cannot provide the foregoing guarantee. By contrast, we design a non-local delegation mechanism that does provably outperform direct voting under mild assumptions about voters.
\end{abstract}

\section{Introduction}

Liquid democracy is a modern approach to voting in which voters can either vote directly or delegate their vote to other voters. In contrast to the classic proxy voting paradigm (Miller, 1969), the key innovation underlying liquid democracy is that proxies - who were selected by voters to vote on their behalf - may delegate their own vote to a proxy, and, in doing so, further delegate all the votes entrusted to them. Put another way (to justify the liquid metaphor), votes may freely flow through the directed delegation graph until they reach a sink, that is, a vertex with outdegree 0 . When the election takes place, each voter who did not delegate her vote is weighted by the total number of votes delegated to him, including her own. In recent years, this approach has been implemented and used on a large scale, notably by eclectic political parties such as the German Pirate Party (Piratenpartei) and Sweden's Demoex (short for Democracy Experiment).

One reason for the success of liquid democracy is that it is seen as a practical compromise between direct democracy (voters vote directly on every issue) and representative democracy, and, in a sense, is the best of both worlds. Direct democracy is particularly problematic, as nicely articulated by Green-Armytage (2015): 
"Even if it were possible for every citizen to learn everything they could possibly know about every political issue, people who did this would be able to do little else, and massive amounts of time would be wasted in duplicated effort. Or, if every citizen voted but most people did not take the time to learn about the issues, the results would be highly random and/or highly sensitive to overly simplistic public relations campaigns."

By contrast, under liquid democracy, voters who did not invest an effort to learn about the issue at hand (presumably, most voters) would ideally delegate their votes to well-informed voters. This should intuitively lead to collective decisions that are less random, and more likely to be correct, than those that would be made under direct democracy.

Our goal is to rigorously investigate the intuition that liquid democracy "outperforms" direct democracy from an algorithmic viewpoint. Indeed, we are interested in delegation mechanisms, which decide how votes should be delegated based on how relatively informed voters are, and possibly even based on the structure of an underlying social network. Our main research question is:

Are there delegation mechanisms that are guaranteed to yield more accurate decisions than direct voting?

\subsection{Overview of the Model and Results}

We focus on a (common) setting where a decision is to be made on a binary issue, i.e., one of two alternatives must be selected (see Section 6 for a discussion of the case of more than two alternatives). To model the idea of accuracy, we assume that one alternative is correct, and the other is incorrect. Each voter $i$ has a competence level $p_{i}$, which is the probability he would vote correctly if he cast a ballot himself.

To motivate this modeling assumption, consider the case of a country deciding between two potential economic policies with the goal of making the country as a whole prosperous. If we pick a measure by which to evaluate these policies (e.g., GDP in ten years), one policy will be objectively better than the other.

Voters may delegate their votes to neighbors in a social network, represented as a directed graph. At the heart of our model is the assumption that voters may only delegate their votes to strictly more competent neighbors (and, therefore, there can be no delegation cycles). Specifically, we say that voter $i$ approves voter $j$ if $p_{j}>p_{i}+\alpha$, for a parameter $\alpha \geq 0$; voters may only delegate to approved neighbors. In defense of this strong assumption, we note that the first of our two theorems - arguably the more interesting of the two - is an impossibility result, so assuming that delegation necessarily boosts accuracy only strengthens it.

As mentioned above, we are interested in studying delegation mechanisms, which decide how votes are delegated (possibly randomly), based on the underlying graph and the approval relation between voters. We pay special attention to local delegation mechanisms, which make delegation decisions based only on the neighborhood of each voter. Local mechanisms capture the spirit of liquid democracy in that voters make independent delegation deci- 
sions based solely on their own viewpoint, without guidance from a central authority. By contrast, non-local mechanisms intuitively require a centralized algorithm that coordinates delegations.

Recall that our goal is to design delegation mechanisms that are guaranteed to be more accurate than direct voting. To this end, we define the gain of a mechanism with respect to a given instance as the difference between the probability that it makes a correct decision (when votes are delegated and weighted majority voting is applied) and the probability that direct voting makes a correct decision on the same instance. The desired guarantee can be formalized via two properties of mechanisms: positive gain ( $P G)$, which means that there are some sufficiently large instances in which the mechanism has positive gain that is bounded away from 0; and do no harm (DNH), which requires that the loss (negative gain) of the mechanism goes to 0 as the number of voters grows. These properties are both weak; in particular, $\mathrm{PG}$ is a truly minimal requirement which, in a sense, mainly rules out direct voting itself as a delegation mechanism.

In Section 4, we study local delegation mechanisms and establish an impossibility result: such mechanisms cannot satisfy both PG and DNH. In a nutshell, the idea is that for any local delegation mechanism that satisfies PG we can construct an instance where few voters amass a large number of delegated votes, that is, delegation introduces significant correlation between the votes. The instance is such that, when the high-weight voters are incorrect, the weighted majority vote is incorrect; yet direct voting is very likely to lead to a correct decision.

In Section 5, we show that non-local mechanisms can circumvent the foregoing impossibility. Specifically, we design a delegation mechanism, GREEDYCAP, that satisfies the PG and DNH properties under mild assumptions about voter competencies. It does so by imposing a cap on the number of votes that can be delegated to any particular voter, thereby avoiding excessive correlation.

In conclusion, our work highlights the significance, and potential dangers, of delegating many votes to few voters. Importantly, there is evidence that this can happen in practice. For example, Der Spiegel reported ${ }^{1}$ that one member of the German Pirate Party, a linguistics professor at the University of Bamberg, amassed so much weight that his "vote was like a decree." Although recent work by Kling et al. (2015) highlights the fact that, in practice, high-weight voters vote reasonably and do not abuse their power, our results corroborate the intuition that this situation should ideally be avoided.

\section{Related Work}

We first present related work that appeared before the publication of the conference version of this paper (Kahng, Mackenzie, \& Procaccia, 2018), then discuss papers that appeared subsequently.

1. http://www.spiegel.de/international/germany/liquid-democracy-web-platform-makes-professor-mostpowerful-pirate-a-818683.html 


\subsection{Previous Related Work}

There is a significant body of work on delegative democracy and proxy voting (Miller, 1969; Tullock, 1992; Alger, 2006). In particular, Cohensius et al. (2017) study a model where voters' positions on an issue are points in a metric space. In their version of direct democracy, a small subset of active voters report their positions, and an aggregation method (such as the median or mean when the metric space is the real line) outputs a single position. Under proxy voting, each inactive voter delegates her vote to the closest active voter. Cohensius et al. identify conditions under which proxy voting gives a more accurate outcome than direct voting, where the measure is proximity of the outcome to the aggregation method applied to all voters' positions.

To the best of our knowledge, there are only two papers prior to the initial publication of our work that provide theoretical analyses of liquid democracy. The first is a paper by Green-Armytage (2015). He considers a setting where, similarly to Cohensius et al. (2017), voters are identified with points on the real line, but in his model votes are noisy estimates of those positions. Green-Armytage defines the expressive loss of a voter as the squared distance between her vote and her position and proves that delegation (even transitive delegation) can only decrease the expressive loss in his model. He also defines systematic loss as the squared distance between the median vote and the median position, but discusses this type of loss only informally (interestingly, he does explicitly mention that correlation can lead to systematic loss in his model).

The second paper is by Christoff and Grossi (2017). They introduce a model of liquid democracy based on the theory of binary aggregation (i.e., their model has a mathematical logic flavor). Their results focus on two problems: the possibility of delegation cycles, and logical inconsistencies that can arise when opinions on interdependent propositions are expressed through proxies. Neither of these issues appears in our model (although the need to avoid cycles is certainly a concern in practice).

Further afield, there is a rich body of work in computational social choice (Brandt, Conitzer, Endriss, Lang, \& Procaccia, 2016) on the aggregation of objective opinions. As in our work, the high-level goal is to pinpoint the correct outcome based on noisy votes. However, previous work in this area does not encompass any notion of vote delegation.

One seminal result in the aggregation of objective opinions - in particular, when deciding between two options, one of which is correct and the other of which is incorrect - is the Condorcet Jury Theorem (Grofman, Owen, \& Feld, 1983), which states that if voters are independent and each have probability greater than $1 / 2$ of choosing the correct outcome, then the probability of choosing correctly approaches one as the size of the electorate increases. Note that the Condorcet Jury Theorem is directly applicable to the setting of direct democracy, but not immediately to the (weighted) setting of liquid democracy. 


\subsection{Subsequent Related Work}

Following the initial publication of our work in AAAI 2018, several papers have explored the theoretical foundations of liquid democracy.

Notably, a paper by Gölz et al. (2018) studies the question of reducing the maximum weight of any voter in liquid democracy systems by permitting voters to specify multiple delegation options and then allowing a mechanism to resolve these delegations. Under a probabilistic model of delegation behavior based on preferential attachment, they establish a doublyexponential asymptotic separation between the expected maximum weight of a voter in the single delegation and multiple delegation settings. Furthermore, they present empirical results that corroborate the large reduction in maximum weight for a realistic number of voters. In contrast to the present paper, their setting does not assume the existence of an objective ground truth, and they do not consider the probability of the electorate coming to the "right" decision; rather, they focus on decreasing the maximum weight of any voter in the system, which is a problem faced by liquid democracy systems in practice (Kling et al., 2015).

Another paper by Brill and Talmon (2018) considers liquid democracy in the setting of ordinal elections in which the electorate wishes to construct a complete ordering over alternatives, as opposed to deciding a binary issue as in this work. In this framework, each voter may specify a partial ordering over the alternatives and delegate to others in order to construct a complete ranking. However, decisions made by delegates may violate transitivity with respect to each voter's partial ordering, and even checking whether delegated votes satisfy transitivity is NP-hard. In order to circumvent issues of transitivity, they introduce a novel class of voting rules for liquid democracy based on distance rationalization, which take as input (perhaps intransitive) delegation graphs and output the "closest" consensus profile.

Bloembergen et al. (2019) consider a game-theoretic version of liquid democracy in which voters must determine whether or not it is rational to delegate their votes to others. They introduce a delegation game in which each voter has a hidden true "type" that she knows imperfectly, and the goal of each voter is to communicate her true type to the mechanism either directly (by voting) or indirectly (by delegating). While this setting distills the problem of finding delegates that represent one's own opinion, it focuses on proving the existence of Nash equilibria under certain assumptions and provides only weak performance bounds in the setting we consider.

Additionally, Abramowitz and Mattei (2018) propose a variant of representative democracy that incorporates ideas of liquid democracy by allowing voters to alter the voting weights of their representatives depending on the issue at hand. Although this circumvents some issues of liquid democracy - for instance, because delegations are no longer transitive, delegation cycles cannot occur - the proposed system is considerably more constrained than general liquid democracy.

Finally, Caragiannis and Micha (2019) extend the model proposed by the conference version of this work and prove further impossibility results. In particular, they show that liquid 
democracy can be much worse than either direct democracy or a complete dictatorship, and they also demonstrate that the problem of optimally coordinating delegations in a centralized manner in order to maximize the probability of discovering the ground truth is NP-hard.

\section{The Model}

We represent an instance of our problem using a directed, labeled graph $G=(V, E, \vec{p})$. $V=\{1, \ldots, n\}$ is a set of $n$ voters, also referred to as vertices (we use the two terms interchangeably). $E$ represents a (directed) social network in which the existence of an edge $(i, j)$ means that voter $i$ knows (of) voter $j$. We denote the neighborhood of voter $i, N_{G}(i)$, to be the set of neighbors that $i$ knows of. Formally, $N_{G}(i)=\{j \in V: i$ knows of $j\}$.

We assume that the voters vote on a binary issue; there is a correct alternative and an incorrect alternative. Each voter $i \in V$ is labeled by her competence level $p_{i}$. This is the probability that $i$ has the correct opinion about the issue at hand, i.e., the probability that $i$ will vote correctly.

Our setting is also parameterized by $\alpha \in(0,1)$. Given this parameter and a labeled graph $G=(V, E, \vec{p})$, we define an approval relation between voters: $i \in V$ approves $j \in V$ if $(i, j) \in E$ and $p_{j} \geq p_{i}+\alpha$. In words, $i$ approves her neighbor $j$ if the difference in their competence levels is at least than $\alpha$. Note that the approval relation is acyclic because $\alpha>0$. Denote

$$
A_{G}(i)=\{j \in V: i \text { approves } j\}
$$

\subsection{Delegation Mechanisms}

The liquid democracy paradigm is implemented through a delegation mechanism $M$, which takes as input a labeled graph $G$, and outputs, for each voter $i$, a delegation probability distribution over $A_{G}(i) \cup\{i\}$ that represents the probability that $i$ will delegate her vote to each of her approved neighbors, or to himself (which means she does not delegate her vote).

To determine whether a delegation mechanism $M$ makes a correct decision on a labeled graph $G=(V, E, \vec{p})$, we use the following 4-step process (which is described in words to avoid introducing notation that will not be used again):

1. Apply $M$ to $G$ to output a delegation probability distribution for each voter $i$.

2. Sample the probability distribution for each vertex to obtain an acyclic delegation graph. Each sink $i$ of the delegation graph (i.e., vertex with no outgoing edges) has weight equal to the number of vertices with directed paths to $i$, including $i$ itself.

3. Each sink $i$ votes for the correct alternative with probability $p_{i}$, and for the incorrect alternative with probability $1-p_{i}$.

4. A decision is made based on the weighted majority vote. ${ }^{2}$

2. Ties can be broken arbitrarily. 
We denote the probability that the mechanism $M$ makes a correct decision on graph $G$ via this 4-step process by $P_{n}^{M}(G)$.

\subsection{Local Mechanisms}

We are particularly interested in a special class of delegation mechanisms that we call local mechanisms. Intuitively, local mechanisms capture the natural setting where each voter makes an independent delegation decision without central coordination or knowledge of global properties about the delegation graph. Formally, a local delegation mechanism is a delegation mechanism such that the probability distribution of each vertex $i$ depends only on (a) the subset $A_{G}(i)$ of neighbors that $i$ approves, (b) an arbitrary ranking $\pi_{i}$ over $A_{G}(i)$, and (c) $N_{G}(i)$, or $i$ 's neighborhood. Note that the ranking $\pi_{i}$ does not have any inherent meaning; it is simply a way to distinguish specific neighbors. In particular, local mechanisms assume that each voter has knowledge of the identities of her approved and non-approved neighbors; a local delegation mechanism is applied to $\pi_{i}$ and $N_{G}(i)$ in order to output a delegation probability distribution for voter $i$.

For instance, say that in a setting with $\alpha=0.15$, Alice $\left(p_{\text {Alice }}=0.6\right)$ has four neighbors: Bob $\left(p_{\text {Bob }}=0.8\right)$, Carla $\left(p_{\text {Carla }}=0.9\right)$, Dean $\left(p_{\text {Dean }}=0.5\right)$, and Evelyn $\left(p_{\text {Evelyn }}=0.7\right)$. Alice approves of Bob and Carla, and let $\pi_{\text {Alice }}=$ Carla $\succ$ Bob. Then, the local delegation mechanism takes $\pi_{\text {Alice }}$ and the set of Alice's neighbors, and returns a probability distribution over delegating to Bob, delegating to Carla, and voting directly.

Let us give some examples of local delegation mechanisms:

- Voters do not delegate their votes. This direct voting mechanism plays a special role in our model, and we denote it by $D$.

- Each voter delegates her vote to a random approved neighbor, if she has any.

- Each voter delegates her vote to a random approved neighbor, if she has approved neighbors but has even more non-approved neighbors.

- Each voter delegates her vote deterministically to a single approved neighbor (e.g., the first in her local ordering $\pi_{i}$ ), if she has any. The ranking $\pi_{i}$ is needed only in order to enable this type of mechanism.

By contrast, the following delegation mechanisms are not local:

- Each voter delegates her vote to her most competent approved neighbor. (Voters cannot distinguish between their approved neighbors, except through the information given by the "arbitrary" ranking $\pi_{i}$.)

- Let there exist a distinguished voter with global identifier $V_{1}$. If $V_{1}$ appears in the approval set of any voter, that voter delegates to $V_{1}$ with probability 1 .

- Each voter delegates her vote only if all agents in her approval set have global identifiers that are even integers. 
- Each voter delegates her vote to an approved neighbor who does not, in turn, delegate her vote to anyone else.

\subsection{Desiderata}

Recall that we are interested in comparing the likelihood of making correct decisions via delegative voting with that of direct voting. To this end, define the gain of delegation mechanism $M$ on labeled graph $G$ as

$$
\operatorname{gain}(M, G)=P_{n}^{M}(G)-P_{n}^{D}(G) .
$$

We would like to design delegation mechanisms that have positive gain (bounded away from zero) in some situations, and which never lose significantly to direct voting. Formally, we are interested in the following two desirable axioms:

- A mechanism $M$ satisfies the positive gain $(P G)$ property if there exist $\gamma>0, n_{0} \in \mathbb{N}$ such that for all $n \geq n_{0}$ there exists a graph $G_{n}$ on $n$ vertices $\operatorname{such}$ that gain $\left(M, G_{n}\right) \geq$ $\gamma$.

- A mechanism $M$ satisfies the do no harm (DNH) property if for all $\varepsilon>0$, there exists $n_{1} \in \mathbb{N}$ such that for all graphs $G_{n}$ on $n \geq n_{1}$ vertices, gain $\left(M, G_{n}\right) \geq-\varepsilon$.

The choice of quantifiers here is of great significance. PG asks for the existence of (large enough) instances where the gain is at least $\gamma$, for a constant $\gamma$. By contrast, DNH essentially requires that any loss would go to 0 as the size of the graph goes to infinity. That is, there may certainly be small instances where delegative voting loses out to direct voting, but that should not be the case in the large.

We note that PG and DNH are defined over worst-case instances. Another natural question to ask is about the expected gain of delegation mechanisms: For a random graph and choice of competence levels, is a given mechanism expected to outperform direct voting? However, we leave this to future work.

\section{Impossibility for Local Mechanisms}

In our model, we make the strong assumption that voters can only delegate their vote to other voters who are more competent than they are, and, in particular, delegation chains can significantly boost the competence of any particular vote. Under this assumption, it seems natural to expect that delegative voting will always do at least as well as direct voting in every situation, and strictly better in some situations. This should intuitively be true under local mechanisms, say, when each voter delegates her vote to an arbitrary approved neighbor (if she has any). The following example helps build intuition for what can go wrong.

Example 1. Consider the labeled graph $G_{n}=(V, E, \vec{p})$ over $n$ vertices, where $E=\{(i, 1)$ : $i \in V \backslash\{1\}$, i.e., $G$ is a star with 1 at the center. Moreover, $p_{1}=4 / 5, p_{i}=2 / 3$ for all $i \in V \backslash\{1\}$, and $\alpha=1 / 10$. Then, as $n$ grows larger, $P_{n}^{D}\left(G_{n}\right)$ goes to 1 by the Law of 
Large Numbers, or, equivalently, by the Condorcet Jury Theorem (Grofman et al., 1983). By contrast, all leaves approve the center, and a naïve local delegation mechanism $M$ would delegate all their votes. In that case, the decision depends only on the vote of the center, so $P_{n}^{M}\left(G_{n}\right)=4 / 5$ for all $n \in \mathbb{N}$, and gain $\left(M, G_{n}\right)$ converges to $-1 / 5$. We conclude that $M$ violates the $\mathrm{DNH}$ property.

One might hope that there are "smarter" local delegation mechanisms, that, say, recognize that when a voter only has one approved neighbor, her vote should not be delegated. However, our first result shows that this is not the case: local delegation mechanisms cannot even satisfy the two minimal requirements of PG and DNH.

Theorem 1. For any $\alpha_{0} \in(0,1)$ such that $i \in V$ approves $j \in V$ if $(i, j) \in E$ and $p_{j}>p_{i}+\alpha_{0}$, there is no local mechanism that satisfies the $P G$ and DNH properties.

The first step in the proof is to better understand the way in which local mechanisms are constrained. This is captured by the following lemma.

Lemma 1. Let $M$ be a local mechanism. Then $M$ satisfies the $P G$ property only if there exist $k, m, \rho>0$ such that, if a voter approves $k$ out of her $m$ total neighbors, then the total probability of delegation to any of these approved neighbors is exactly $\rho$.

Proof. Suppose that PG holds. Let $\gamma>0$ and fix a labeled graph $G$ such that $\operatorname{gain}(M, G) \geq$ $\gamma>0$. In order for this to be the case, there must exist some vertex $i$ that delegates with positive probability $\rho$. Let $k$ be the number of neighbors in $G$ that $i$ approves, and let $m$ be her total number of neighbors in $G$; this yields the desired tuple $(k, m, \rho) .^{3}$

The crux of the theorem's proof is the construction of a graph that, from the local viewpoint of many of the vertices, looks like the neighborhood prescribed by Lemma 1. Specifically, a $k$-center m-uniform star consists of vertices called leaves that are each connected to $k$ central vertices (the centers) as well as $m-k$ other leaves. Each leaf vertex has competence level $p_{\ell}$, and each center vertex has competence level $p_{c}$, such that $p_{c}>p_{\ell}+\alpha$. We set the value of $k$ and $m$ to be the values whose existence is guaranteed by Lemma 1, which means that the construction of a $k$-center $m$-uniform star satisfies the property that each leaf delegates to some center vertex with probability $\rho$. Throughout the proof, we will let $n_{c}=k$ be the number of centers, and $n_{\ell}$ will denote the number of leaves.

At a high level, we show that the loss of any local mechanism can approach $\left(1-p_{c}\right)^{k}$, which is constant given $k$. We do this by constructing a graph that consists of a $k$-center $m$-uniform star with an independent disconnected component consisting of $n_{d}$ vertices of competence level $p_{d}$. We set the parameters so that the direct voting mechanism $D$ decides correctly with high probability. By contrast, under the local delegation mechanism $M$, enough leaves delegate their votes to the centers so that if all centers were to vote incorrectly, which happens with probability $\left(1-p_{c}\right)^{k}$, then $M$ would decide incorrectly. While the basic idea is simple enough, the formal construction is quite delicate, as many different parameters must be carefully balanced.

3. Note that the conclusion is invariant to the ranking $\pi_{i}$. 


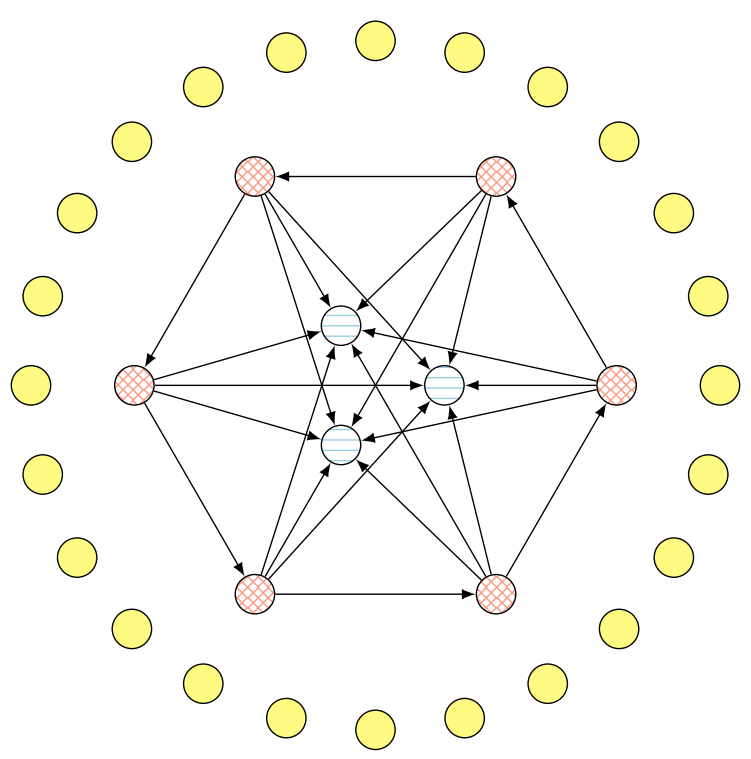

Figure 1: Graph $G$ for $n_{\ell}=6$ leaves (shown in crosshatched red), $n_{c}=3$ centers (shown in striped blue), $n_{d}=24$ disconnected vertices (shown in solid yellow), and $m=4$.

Proof of Theorem 1. Let $M$ be a local mechanism that satisfies PG. By Lemma 1, there must exist at least one $(k, m, \rho)$ tuple for $M$ that satisfies the lemma's conclusion. For any $n_{1}$ prescribed by DNH and any $\alpha_{0}$, we can construct a graph $G_{n}$ with $n \geq n_{1}$ such that DNH does not hold.

Let $G$ be a graph of size $n=n_{c}+n_{\ell}+n_{d}$, where $n_{c}=k$, that consists of a $k$-center $m$-uniform star and a disconnected component containing $n_{d}$ disconnected points (see Figure 1). Each center has competence level $p_{c}$, each leaf in the star has competence level $p_{\ell}$, and each point in the disconnected component has competence level $p_{d}$. Given $(k, m, \rho), n_{1}$, and $\alpha_{0}$, note that the following constraints must hold.

$$
\begin{aligned}
n_{\ell} & \geq m-n_{c} \\
n & =n_{\ell}+n_{c}+n_{d} \geq n_{1} \\
p_{c} & >p_{\ell}+\alpha_{0}
\end{aligned}
$$

We will prove that the construction above instantiated with the following parameter values violates DNH for any input of $(k, m, \rho), n_{1}$, and $\alpha=\alpha_{0}+\varepsilon^{\prime}$ for $\varepsilon^{\prime}=\frac{1-\alpha_{0}}{2}>0$, for sufficiently small $\delta$ (i.e., as $\delta \rightarrow 0$ ).

We begin by defining the sizes of each component: $n_{c}, n_{\ell}$, and $n_{d}$.

$$
\begin{aligned}
n_{c} & =k \\
n_{\ell} & =\frac{n_{1} m}{\alpha \delta} \\
n_{d} & =C_{1} \frac{n_{1} m}{\alpha \delta}
\end{aligned}
$$


Note that $n_{d}$ depends on a constant $C_{1}$, which, along with another constant $\sigma$, is defined next.

$$
\begin{aligned}
C_{1} & =\frac{\left(\frac{\left(\frac{p_{\ell} \rho}{\sigma} n_{\ell}-p_{\ell} \sqrt{n_{\ell}}\right)}{2}\right)^{2}-n_{c}}{n_{\ell}}-1 \\
\sigma & =\sqrt{-\frac{\ln \left(\frac{\delta}{2}\right)}{2}}^{n^{2}}
\end{aligned}
$$

Now, we define the competency values for each component, $p_{c}, p_{\ell}$, and $p_{d}$. Note that there is a range of acceptable compentency values for $p_{d}$.

$$
\begin{aligned}
& p_{c}=\frac{1+\alpha}{2} \\
& p_{\ell}=\frac{1-\alpha}{2} \\
& p_{d} \in\left[\left(\frac{n / 2-n_{\ell} p_{\ell}}{n_{d}}\right)+\frac{\sigma \sqrt{n}}{n_{d}},\left(\frac{n / 2-n_{\ell} p_{\ell}}{n_{d}}\right)+\frac{\left(n_{\ell} \rho-\tau\right) p_{\ell}-\sigma \sqrt{n}}{n_{d}}\right]
\end{aligned}
$$

Finally, we define $\tau$, another constant that will be useful for establishing concentration guarantees in the proof.

$$
\tau=\sqrt{-\frac{\left(\ln \frac{\delta}{2}\right) n_{\ell}}{2}}
$$

The following claim asserts that the construction is feasible.

Claim 1. $C_{1}>0$ and the range of values for $p_{d}$ in (11) is nonempty.

Proof. From (7), we have

$$
C_{1}=\frac{\left(\frac{\left(\frac{p_{\ell} \rho}{\sigma} n_{\ell}-p_{\ell} \sqrt{n_{\ell}}\right)}{2}\right)^{2}-k}{n_{\ell}}-1
$$

and rearranging terms yields

$$
2 \sqrt{\left(C_{1}+1\right) n_{\ell}+k}=\frac{p_{\ell} \rho}{\sigma} n_{\ell}-p_{\ell} \sqrt{n_{\ell}} .
$$

Now, note that $n_{d}=C_{1} n_{\ell}$ and therefore $\left(C_{1}+1\right) n_{\ell}+k=n_{d}+n_{\ell}+k=n$. Additionally, note that $\sqrt{n_{\ell}}=\frac{\tau}{\sigma}$. Substituting this in, we have

$$
2 \sqrt{n}=\frac{p_{\ell} \rho n_{\ell}-p_{\ell} \tau}{\sigma}
$$

and therefore

$$
\sigma \sqrt{n}=p_{\ell}\left(\rho n_{\ell}-\tau\right)-\sigma \sqrt{n} .
$$


Now, we note that $\sigma \sqrt{n}-k p_{c}<\sigma \sqrt{n}$ and

$$
\sqrt{n}>\sqrt{n-k-\left(\rho n_{\ell}-\tau\right)}
$$

because both $k$ and $\rho n_{\ell}-\tau=2 \sigma \sqrt{n} / p_{\ell}$ are greater than 0 , and $n-k-\left(\rho n_{\ell}-\tau\right)>$ $n-k-n_{\ell} \geq 0$. Now, from (13), we can conclude that

$$
\begin{aligned}
\sigma \sqrt{n}-k p_{c} & <\sigma \sqrt{n}=p_{\ell}\left(\rho n_{\ell}-\tau\right)-\sigma \sqrt{n} \\
& <\left(\rho n_{\ell}-\tau\right) p_{\ell}-\sigma \sqrt{n-k-\left(\rho n_{\ell}-\tau\right)}
\end{aligned}
$$

which means

$$
p_{d} \in\left[\left(\frac{n / 2-n_{\ell} p_{\ell}}{n_{d}}\right)+\frac{\sigma \sqrt{n}-k p_{c}}{n_{d}},\left(\frac{n / 2-n_{\ell} p_{\ell}}{n_{d}}\right)+\frac{\left(n_{\ell} \rho-\tau\right) p_{\ell}-\sigma \sqrt{n-k-\left(n_{\ell} \rho-\tau\right)}}{n_{d}}\right]
$$

is non-empty, and our value for $p_{d}$ is admissible.

Lastly, we have to show that $C_{1}$ is itself admissible; i.e., that the following holds:

$$
\frac{\left(\frac{\left(\frac{p_{\ell} \rho}{\sigma} n_{\ell}-p_{\ell} \sqrt{n_{\ell}}\right)}{2}\right)^{2}-k}{n_{\ell}}-1>0 .
$$

Rearranging and expanding, we obtain

$$
\frac{p_{\ell} n_{\ell} \rho}{\sigma}-p_{\ell} \sqrt{n_{\ell}} \geq 2 \sqrt{n_{\ell}+k} .
$$

Now, note that both sides are positive as $\delta \rightarrow 0$. Indeed, the right hand side consists of positive terms and the left hand side simplifies to $p_{\ell} \sqrt{n_{\ell}}\left(\rho \sqrt{n_{\ell}} / \sigma-1\right)$, which is positive iff $\rho \sqrt{n_{\ell}}>\sigma$, which is true as $\delta \rightarrow 0$ because $1 / \delta$ grows more quickly than $\ln (2 / \delta)$. Therefore, squaring both sides yields

$$
\left(\frac{p_{\ell} n_{\ell} \rho}{\sigma}\right)^{2}+\left(p_{\ell}\right)^{2} n_{\ell}-2 \frac{\left(p_{\ell}\right)^{2} \rho\left(n_{\ell}\right)^{3 / 2}}{\sigma} \geq 4\left(n_{\ell}+k\right) .
$$

Now, substituting in our value for $n_{\ell}$, we obtain

$$
\left[\left(\frac{p_{\ell} \rho}{\sigma}\right)\left(\frac{n_{1} m}{\alpha \delta}\right)\right]^{2}+\left(p_{\ell}\right)^{2}\left(\frac{n_{1} m}{\alpha \delta}\right)-\frac{2\left(p_{\ell}\right)^{2} \rho}{\sigma}\left(\frac{n_{1} m}{\alpha \delta}\right)^{3 / 2}-4\left(\frac{n_{1} m}{\alpha \delta}\right)-4 k
$$

As $\delta \rightarrow 0$, (14) becomes dominated by the highest-order $1 / \delta$ term, and therefore is always positive for any assignment to the other variables because the rest of them are constrained to be strictly positive.

Because $\alpha, \delta \in(0,1)$, the value of $n_{\ell}$ in (5) is greater than both $n_{1}$ and $m$, hence constraints (1) and (2) are immediately satisfied. Moreover, constraint (3) is satisfied by (9) and (10).

Turning to the proof that DNH is violated, let $\operatorname{cor}_{D}$, $\operatorname{del}_{M}$, and nondel $_{M}$ be the random variables corresponding to the number of correct votes under $D$, the number of delegated 
correct votes under $M$, and the number of non-delegated correct votes under $M$. Additionally, let $\varepsilon, \tau$ (recalled again for convenience), and $\xi$ be as follows.

$$
\begin{aligned}
\varepsilon & =\sqrt{-\frac{\left(\ln \frac{\delta}{2}\right) n}{2}}, \\
\tau & =\sqrt{-\frac{\left(\ln \frac{\delta}{2}\right) n_{\ell}}{2}}, \text { and } \\
\xi & =\sqrt{-\frac{\left(\ln \frac{\delta}{2}\right)\left(n-n_{c}-\left(\rho n_{\ell}-\tau\right)\right)}{2}} .
\end{aligned}
$$

Our goal is to bound the expectations of $\operatorname{cor}_{D}$, $\operatorname{del}_{M}$, and $\operatorname{nondel}_{M}$. First, we examine $\mathbb{E}\left[\operatorname{cor}_{D}\right]$. We would like to show that

$$
\mathbb{E}\left[\operatorname{cor}_{D}\right] \geq n / 2+\varepsilon .
$$

Expanding out the expected value, this is equivalent to

$$
p_{c} n_{c}+p_{\ell} n_{\ell}+p_{d} n_{d} \geq n / 2+\varepsilon .
$$

From (11), we have

$$
p_{d} \geq \frac{n / 2-p_{\ell} n_{\ell}+\varepsilon}{n_{d}},
$$

so it is sufficient to show that

$$
p_{c} n_{c}+p_{\ell} n_{\ell}+n_{d}\left(\frac{n / 2-p_{\ell} n_{\ell}+\varepsilon}{n_{d}}\right) \geq n / 2+\varepsilon,
$$

and simplifying this expression yields $p_{c} n_{c} \geq 0$. This is true by Equation (9), because $\alpha$ and $k$ are both constrained to be strictly positive.

Next, we examine $\mathbb{E}\left[\operatorname{del}_{M}\right]$. We would like to show that

$$
\mathbb{E}\left[\operatorname{del}_{M}\right]=n_{\ell} \rho .
$$

This is trivial to see, as $\operatorname{del}_{M}$ is a sum of $n_{\ell}$ Bernoulli random variables with "success" probability $\rho$.

Finally, we examine the "typical case" over nondel $_{M}$, or $\mathbb{E}\left[\right.$ nondel $\left._{M} \mid \operatorname{del}_{M}=v\right]$ for all integers $v \in\left[n_{\ell} \rho-\tau, n_{\ell} \rho+\tau\right]$. Intuitively, this case considers the number of correct votes cast by still-independent vertices after "enough" leaf vertices have delegated their votes. If these votes do not make up a majority, then all centers voting incorrectly will cause the entire graph to vote incorrectly. We would like to show that

$$
\mathbb{E}\left[\operatorname{nondel}_{M} \mid \operatorname{del}_{M}=v\right] \leq n / 2-\xi
$$

for all integers $v \in\left[n_{\ell} \rho-\tau, n_{\ell} \rho+\tau\right]$. Conditionally on $d_{e} l_{M}$ being in the prescribed range above, we see that in the worst case, $\operatorname{del}_{M}=n_{\ell} \rho-\tau$, meaning the fewest possible voters delegate under this assumption. Given this, we would like to show that

$$
p_{d} n_{d}+p_{\ell}\left(n_{\ell}-\left(\rho n_{\ell}-\tau\right)\right) \leq n / 2-\xi .
$$


From Equation (11) we have

$$
p_{d} \leq \frac{n / 2-p_{\ell} n_{\ell}+\left(n_{\ell} \rho-\tau\right) p_{\ell}-\xi}{n_{d}}
$$

which yields

$$
\begin{gathered}
\left(\frac{n / 2-p_{\ell} n_{\ell}+\left(n_{\ell} \rho-\tau\right) p_{\ell}-\xi}{n_{d}}\right) n_{d}+ \\
p_{\ell}\left(n_{\ell}-\left(\rho n_{\ell}-\tau\right)\right) \\
\leq n / 2-\xi
\end{gathered}
$$

Simplifying yields $0 \leq 0$ - a tautology. This establishes Equation (17).

We now wish to bound the probability of $\operatorname{cor}_{D}, \operatorname{del}_{M}$, and nondel $_{M}$ deviating by too much. We use Hoeffding's inequality (Hoeffding, 1963), which states that given $n$ independent Bernoulli random variables $X_{i} \in[0,1]$ and $X=\sum_{i} X_{i}$, the following concentration bound holds:

$$
\operatorname{Pr}[|X-\mathbb{E}[X]| \geq \varepsilon] \leq 2 \exp \left(\frac{-2 \varepsilon^{2}}{n}\right)
$$

First, we examine $\operatorname{cor}_{D}$. From (18) and a straightforward substitution for $\varepsilon$, we obtain

$$
\begin{aligned}
\operatorname{Pr}\left(\left|\operatorname{cor}_{D}-\mathbb{E}\left[\operatorname{cor}_{D}\right]\right| \geq \varepsilon\right) & \leq 2 \exp \left(\frac{-2 \varepsilon^{2}}{n}\right) \\
& =2 \exp \left(-\frac{2\left[\sqrt{\left.-\frac{\left(\ln \frac{\delta}{2}\right) n}{2}\right]^{2}}\right.}{n}\right) \\
& =\delta .
\end{aligned}
$$

Likewise, for $\operatorname{del}_{M}$, from (18) and a straightforward substitution for $\tau$, we obtain

$$
\begin{aligned}
\operatorname{Pr}\left[\left|d e l_{M}-\mathbb{E}\left[\operatorname{del}_{M}\right]\right| \geq \tau\right] & \leq 2 \exp \left(\frac{-2 \tau^{2}}{n_{\ell}}\right) \\
& =2 \exp \left(-\frac{2\left[\sqrt{-\frac{\left(\ln \frac{\delta}{2}\right) n_{\ell}}{2}}\right]^{2}}{n_{\ell}}\right) \\
& =\delta .
\end{aligned}
$$

Finally, for $\operatorname{nondel}_{M}$, we are interested in upper-bounding

$$
\operatorname{Pr}\left[\mid \text { nondel }_{M}-\mathbb{E}\left[\text { nondel }_{M} \mid \text { del }_{M}=v\right]|\geq \xi| \text { del }_{M}=v\right]
$$


for every integer $v \in\left[n_{\ell} \rho-\tau, n_{\ell} \rho+\tau\right]$. As before, we apply Equation (18), and, as it turns out, we can derive an upper bound when $\operatorname{del}_{M}=n_{\ell} \rho-\tau$. Therefore, we obtain that for every $v \in\left[n_{\ell} \rho-\tau, n_{\ell} \rho+\tau\right]$,

$$
\begin{aligned}
& \operatorname{Pr}\left[\mid \text { }_{\text {ondel }}-\mathbb{E}\left[\text { nondel }_{M} \mid \text { del }_{M}=v\right]|\geq \xi| \text { del }_{M}=v\right] \\
& \quad \leq 2 \exp \left(\frac{-2 \xi^{2}}{n-n_{c}-\left(\rho n_{\ell}-\tau\right)}\right) \\
& \quad=2 \exp \left(-\frac{2\left[\sqrt{-\frac{\left(\ln \frac{\delta}{2}\right)\left(n-n_{c}-\left(\rho n_{\ell}-\tau\right)\right)}{2}}\right)^{2}}{n-n_{c}-\left(\rho n_{\ell}-\tau\right)}\right) \\
& \quad=\delta
\end{aligned}
$$

where the denominator comes from the (worst-case) total number of non-delegated votes under $M$.

From the above, we see that

$$
\begin{aligned}
\operatorname{Pr}\left[\operatorname{cor}_{D}>n / 2\right] & \geq 1-\delta, & & \text { (by (15) and (19)) } \\
\operatorname{Pr}\left[\text { del }_{M} \in\left(n_{\ell} \rho-\tau, n_{\ell} \rho+\tau\right)\right] & \geq 1-\delta, & & \text { (by (16) and (20)) } \\
\operatorname{Pr}\left[\text { nondel }_{M}<n / 2 \mid \text { del }_{M}=v\right] & \geq 1-\delta, & & \text { (by (17) and }(21))
\end{aligned}
$$

where the last inequality holds for all integers $v \in\left[n_{\ell} \rho-\tau, n_{\ell} \rho+\tau\right]$.

Therefore, the lower bound on the probability of $D$ deciding correctly is $p_{d}(G) \geq 1-\delta$. We can lower-bound the probability of $M$ deciding incorrectly in order to upper-bound $P_{n}^{M}(G)$. We slightly overload notation and let $M$ be the event that $M$ decides correctly, and $\neg M$ be the event that $M$ decides incorrectly. Moreover, denote by $V$ the event that $\operatorname{del}_{M} \in\left[n_{\ell} \rho-\tau, n_{\ell} \rho+\tau\right]$. By definition, we have

$$
\operatorname{Pr}[\neg M]=\operatorname{Pr}[\neg M \mid V] \operatorname{Pr}[V]+\operatorname{Pr}[\neg M \mid \neg V] \operatorname{Pr}[\neg V],
$$

and because probabilities cannot be negative,

$$
\operatorname{Pr}[\neg M] \geq \operatorname{Pr}[\neg M \mid V] \operatorname{Pr}[V] .
$$

Now, because $\operatorname{Pr}[V] \geq 1-\delta$,

$$
\operatorname{Pr}[\neg M] \geq \operatorname{Pr}[\neg M \mid V](1-\delta) .
$$

Furthermore, we know that $\operatorname{Pr}[\neg M \mid V]$ is also lower-bounded by $\left(1-p_{c}\right)^{n_{c}}(1-\delta)$ because one setting under which $M$ decides incorrectly is exactly when all centers vote incorrectly and ondel $_{M}<n / 2$. It follows that

$$
\operatorname{Pr}[\neg M] \geq\left(1-p_{c}\right)^{n_{c}}(1-\delta)(1-\delta) .
$$

Therefore, taking the complement, we have an upper bound on the probability of $M$ voting correctly of

$$
\operatorname{Pr}[M] \leq 1-\left(1-p_{c}\right)^{n_{c}}(1-\delta)^{2}
$$


and the total loss can be lower-bounded by

$$
(1-\delta)-\left(1-\left(1-p_{c}\right)^{n_{c}}(1-\delta)^{2}\right)=\left(1-p_{c}\right)^{n_{c}}(1-\delta)^{2}-\delta .
$$

As $\delta \rightarrow 0$, this tends to $\left(1-p_{c}\right)^{n_{c}}=\left(1-p_{c}\right)^{k}$, which is constant and bounded away from 0 . We conclude that $M$ violates the DNH property.

We note that even if each voter had access to a ranking of her approved neighbors by competence (which would allow voters to, for instance, delegate to their most competent neighbor), this impossibility still holds because the construction is such that all approved vertices have equal competence.

\section{Possibility for Non-Local Mechanisms}

The main idea underlying Theorem 1 is that liquid democracy can correlate the votes to the point where the mistakes of a few popular voters tip the scales in the wrong direction. As we show in the theorem's proof, this is unavoidable under local delegation mechanisms, which, intuitively, cannot identify situations in which certain voters amass a large number of votes. However, non-local delegation mechanisms can circumvent this issue. Indeed, consider the following delegation mechanism.

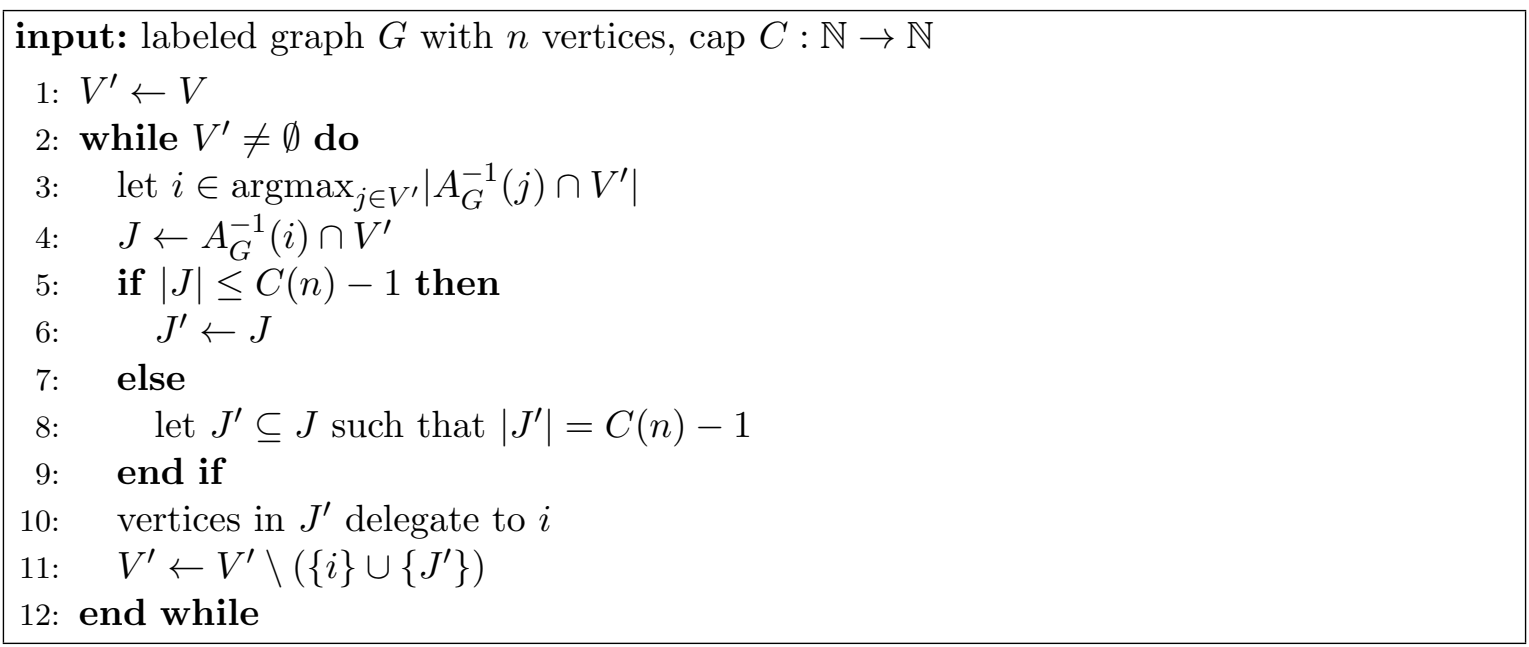

Algorithm 1: GREEDYCAP

In words, the mechanism GREEDYCAP, given as Algorithm 1, receives as input a labeled graph $G$, and a cap $C$ which is a function of $n$. It iteratively selects a voter with maximum approvals, and delegates votes to him, so that no more than $C(n)-1$ votes are delegated to a single voter (that is, no voter can have weight more than $C(n)$ ). All voters involved in the current iteration are then eliminated from further consideration, which is why delegations under this mechanism are only 1-hop.

It is obvious that GreEDyCAP satisfies the PG property. Intuitively, for any value of $\alpha$, it is always possible to construct large instances of graphs where a few voters delegate to more 
competent voters in a way that increases the probability of making the correct decision overall. However, although it seems at first glance that it should satisfy DNH as well (as it solves the excessive correlation problem), the following example shows that, without further assumptions, it does not.

Example 2. Assume for ease of exposition that $\alpha<1 / 3$. For any odd $n=2 k+1$, consider the labeled graph $G_{n}=(V, E, \vec{p})$ on $n$ vertices, defined as follows: $E=\{(1,2)\}$ (i.e., the only edge in the graph is from 1 to 2$), p_{1}=1 / 3, p_{2}=2 / 3$, there are $k$ vertices with $p_{i}=1$, and $k-1$ vertices with $p_{i}=0$. Even if $C(n) \equiv 2$, GREEDYCAP would delegate the vote of voter 1 to voter 2 . Therefore, the mechanism decides correctly if and only if voter 2 votes correctly, which happens with probability $2 / 3$. By contrast, under direct voting, it is enough for either voter 1 or voter 2 to vote correctly, which happens with probability $7 / 9$. It follows that the loss of GreedyCAP is 1/9 - a constant. We conclude that GreedyCaP violates DNH.

The reason the example works is that the outcome completely depends on voters 1 and 2 , as the others vote deterministically (competence level 0 or 1 ). To avoid this problem, we make the natural assumption that competence levels are bounded away from 0 and 1, i.e., voters are never horribly misinformed or perfectly informed. It turns out that this additional assumption is sufficient to guarantee that GREEDYCAP satisfies the DNH property.

Theorem 2. Assume that there exists $\beta \in(0,1 / 2)$ such that all competence levels are in $[\beta, 1-\beta]$. Then for any difference in competencies $\alpha \in(0,1-2 \beta)$, GREEDYCAP with cap $C: \mathbb{N} \rightarrow \mathbb{N}$ such that $C(n) \in \omega(1)$ and $C(n) \in o(\sqrt{\log n})$ satisfies the $P G$ and $D N H$ properties.

We begin with a proof sketch, focusing on the DNH property (as PG is rather simple). Given $n$ voters, we denote the number of correct votes under direct voting and GREEDYCAP by $X_{n}^{D}$ and $X_{n}^{M}$, respectively, and consider two cases.

1. $\left|\mathbb{E}\left[X_{n}^{D}\right]-\frac{n}{2}\right|>\frac{n}{\log n}$.

2. $\left|\mathbb{E}\left[X_{n}^{D}\right]-\frac{n}{2}\right| \leq \frac{n}{\log n}$.

In Case 1 , the direct voting mechanism has mean far away from $n / 2$. When $\mathbb{E}\left[X_{n}^{D}\right]<$ $n / 2-n / \log n$, we can show that $P_{n}^{D}$ goes to 0 as $n$ goes to infinity. This means that DNH is satisfied for any value of $P_{n}^{M}$. In the case where $\mathbb{E}\left[X_{n}^{D}\right]>n / 2+n / \log n$, we can show that $P_{n}^{M}$ goes to 1 as $n$ goes to infinity, which means that DNH is satisfied for any value of $P_{n}^{D}$.

In Case 2 , the direct voting setting has mean close to $n / 2$. From here, we consider two subcases.

1. The number of voters who delegate is greater than $n / g(n)$, where $g(n) \in o(\log n)$ and $g(n) \in \omega\left(C(n)^{2}\right)$ - hence the upper bound on $C(n)$ in the statement of Theorem 2 .

2. The number of voters who delegate is at most $n / g(n)$.

In Subcase 1, because a relatively large fraction of voters delegate their votes to more competent neighbors, $\mathbb{E}\left[X_{n}^{M}\right]-\mathbb{E}\left[X_{n}^{D}\right]$ is large enough to offset the simultaneous increase 
in the variance of $X_{n}^{M}$, and, in the limit, $P_{n}^{M}$ goes to 1 . In Subcase 2, we again have $\mathbb{E}\left[X_{n}^{M}\right] \geq \mathbb{E}\left[X_{n}^{D}\right]$ due to delegation. Additionally, because so few voters delegate, the ratio of the variance of $X_{n}^{M}$ and that of $X_{n}^{D}$ converges to 1 as $n$ approaches infinity, which means that (in the worst case) the difference between $P_{n}^{D}$ and $P_{n}^{M}$ converges to 0 .

Before presenting the theorem's detailed proof, we establish three useful lemmas, as well as definitions needed in the first two lemmas.

Starting with the definitions, we say that a sequence of random variables $\left\{X_{k}\right\}$ is pairwise mixing if for every real $x$,

$$
\sup _{k}\left|\operatorname{Cov}\left[\mathbb{1}\left\{X_{k}<x\right\}, \mathbb{1}\left\{X_{k+n}<x\right\}\right]\right| \rightarrow 0 \text { as } n \rightarrow \infty,
$$

where $\mathbb{1}$ represents an indicator random variable. A collection of random variables $\left\{X_{k}\right\}$ is uniformly integrable if

$$
\sup _{k} \mathbb{E}\left[\left|X_{k}\right| \mathbb{1}\left\{X_{k}>x\right\}\right] \rightarrow 0 \text { as } x \rightarrow \infty
$$

Finally, a sequence of random variables $\left\{X_{k}\right\}$ is a martingale difference sequence if

1. For all $k, \mathbb{E}\left[\left|X_{k}\right|\right]<\infty$, and

2. For all $k, \mathbb{E}\left[X_{k} \mid X_{1}, \ldots, X_{k-1}\right]=0$.

Lemma 2 (Theorem 2.1 in Peligrad and Utev, 1997). Let $X=\left\{X_{1}, \ldots, X_{k}, \ldots\right\}$ be $a$ pairwise mixing martingale difference sequence of random variables, and let $\left\{a_{n k}: 1 \leq k \leq\right.$ $n\}$ be a triangular array of real numbers such that

$$
\begin{aligned}
& \sup _{n} \sum_{k=1}^{n} a_{n k}^{2}<\infty \\
& \max _{1 \leq k \leq n}\left|a_{n k}\right| \rightarrow 0 \text { as } n \rightarrow \infty .
\end{aligned}
$$

Furthermore, assume that $\left\{X_{k}^{2}\right\}$ is a uniformly integrable family and that

$$
\operatorname{Var}\left(\sum_{k=1}^{n} a_{n k} X_{k}\right)=1
$$

Then,

$$
\sum_{k=1}^{n} a_{n k} X_{k} \rightarrow N(0,1) \text { as } n \rightarrow \infty .
$$

The next lemma adapts the previous one for our setting.

Lemma 3. Let $Y=\left\{Y_{1}, \ldots, Y_{n}, \ldots\right\}$ be a sequence of independent Bernoulli random variables where $Y_{i}$ has success probability $p_{i} \in[\beta, 1-\beta]$ for $\beta \in(0,1 / 2)$. Furthermore, define $C(n) \in o\left(n^{1 / 2}\right)$. Now, let $\left\{b_{n k}: 1 \leq k \leq n\right\}$ be a triangular array of nonnegative integers such that $0 \leq b_{n k} \leq C(n)$ for all $1 \leq k \leq n$ and $\sum_{k=1}^{n} b_{n k}=n$. 
Then, $\sum_{k=1}^{n} b_{n k} Y_{k}$ converges to a normal distribution with mean $\sum_{k=1}^{n} b_{n k} \mathbb{E}\left[Y_{k}\right]$ and variance $\sum_{k=1}^{n} b_{n k}^{2} \operatorname{Var}\left[Y_{k}\right]$ as $n$ goes to infinity; i.e.,

$$
\sum_{k=1}^{n} b_{n k} Y_{k} \rightarrow N\left(\sum_{k=1}^{n} b_{n k} \mathbb{E}\left[Y_{k}\right], \sum_{k=1}^{n} b_{n k}^{2} \operatorname{Var}\left[Y_{k}\right]\right) \text { as } n \rightarrow \infty .
$$

Proof. Define $X=\left\{X_{1}, \ldots, X_{n}, \ldots\right\}$ to be a sequence of independent random variables where $X_{i}=Y_{i}-\mathbb{E}\left[Y_{i}\right]$. In other words, $X_{i}$ corresponds to the difference between each $Y_{i}$ and its expectation. Note that $\mathbb{E}\left[X_{i}\right]=0$ for all $i$; i.e., $X$ is a centered sequence, and that $\operatorname{Var}\left[X_{i}\right]=\operatorname{Var}\left[Y_{i}\right]=p_{i}\left(1-p_{i}\right)$.

Let

$$
s_{n}^{2}=\sum_{k=1}^{n} \operatorname{Var}\left[b_{n k} X_{k}\right]=\sum_{k=1}^{n} b_{n k}^{2} \operatorname{Var}\left[X_{k}\right],
$$

and let us define another triangular array of real numbers $\left\{a_{n k}: 1 \leq k \leq n\right\}$ such that $a_{n k}=\frac{b_{n k}}{s_{n}}$. We will show that

$$
\sum_{k=1}^{n} a_{n k} X_{k} \rightarrow N(0,1) \text { as } n \rightarrow \infty
$$

and then use this fact to prove the desired result.

In order to show Equation (24), we wish to apply Lemma 2. To do that, we must make sure that $\left\{X_{k}\right\}$ is a pairwise mixing martingale difference sequence, $\left\{X_{k}^{2}\right\}$ is uniformly integrable, our construction of $\left\{a_{n k}: 1 \leq k \leq n\right\}$ satisfies Equations (22) and (23), and $\operatorname{Var}\left[\sum_{k=1}^{n} a_{n k} X_{k}\right]=1$.

First, note that it is easy to check that $\left\{X_{k}\right\}$ is pairwise mixing, $\left\{X_{k}\right\}$ is a martingale differences sequence, and $\left\{X_{k}^{2}\right\}$ is uniformly integrable because each $X_{k}$ is independent, has expectation 0 , and is bounded in $[-(1-\beta), 1-\beta]$.

Now, we show that $\left\{a_{n k}: 1 \leq k \leq n\right\}$ satisfies Equation (22).

$$
\begin{aligned}
\sup _{n} \sum_{k=1}^{n} a_{n k}^{2} & =\sup _{n} \sum_{k=1}^{n}\left(\frac{b_{n k}}{s_{n}}\right)^{2} \\
& =\sup _{n} \frac{\sum_{k=1}^{n} b_{n k}^{2}}{s_{n}^{2}} \\
& =\sup _{n} \frac{\sum_{k=1}^{n} b_{n k}^{2}}{\sum_{k=1}^{n} b_{n k}^{2} \operatorname{Var}\left[X_{k}\right]} \\
& \left.\leq \sup _{n} \frac{\sum_{k=1}^{n} b_{n k}^{2}}{\beta(1-\beta) \sum_{k=1}^{n} b_{n k}^{2}} \quad \quad \text { (because } \operatorname{Var}\left[X_{k}\right] \geq \beta(1-\beta)\right) \\
& =\frac{1}{\beta(1-\beta)}<\infty
\end{aligned}
$$

because $\beta$ is a constant. 
Next, we show that $\left\{a_{n k}: 1 \leq k \leq n\right\}$ satisfies Equation (23).

$$
\begin{aligned}
\max _{1 \leq k \leq n}\left|a_{n k}\right| & =\max _{1 \leq k \leq n} \frac{b_{n k}}{s_{n}} \\
& =\max _{1 \leq k \leq n} \frac{b_{n k}}{\sqrt{\sum_{k=1}^{n} b_{n k}^{2} \operatorname{Var}\left[X_{k}\right]}} \\
& \leq \frac{C(n)}{\sqrt{\beta(1-\beta) \sum_{k=1}^{n} b_{n k}^{2}}} .
\end{aligned}
$$

Applying Cauchy-Schwarz to the $\sum_{k=1}^{n} b_{n k}^{2}$ term in the denominator yields

$$
\left(\sum_{k=1}^{n} 1^{2}\right)\left(\sum_{k=1}^{n} b_{n k}^{2}\right) \geq\left(\sum_{k=1}^{n} b_{n k}\right)^{2}=n^{2} \Longrightarrow \sum_{k=1}^{n} b_{n k}^{2} \geq n .
$$

Therefore,

$$
\begin{aligned}
\frac{C(n)}{\sqrt{\beta(1-\beta) \sum_{k=1}^{n} b_{n k}^{2}}} & \leq \frac{C(n)}{\sqrt{\beta(1-\beta) n}} \\
& =\frac{1}{\sqrt{\beta(1-\beta)}} \cdot \frac{C(n)}{n^{1 / 2}}
\end{aligned}
$$

Because $\frac{1}{\sqrt{\beta(1-\beta)}}$ is a constant and $C(n) \in o\left(n^{1 / 2}\right)$, we see that this quantity goes to 0 as $n$ approaches infinity, and therefore $\max _{1 \leq k \leq n}\left|a_{n k}\right| \rightarrow 0$ as $n$ approaches infinity.

Lastly, we must check that $\operatorname{Var}\left[\sum_{k=1}^{n} a_{n k} X_{k}\right]=1$. Indeed, doing out the calculation yields

$$
\begin{aligned}
\operatorname{Var}\left[\sum_{k=1}^{n} a_{n k} X_{k}\right] & =\sum_{k=1}^{n} \operatorname{Var}\left[a_{n k} X_{k}\right] \\
& =\sum_{k=1}^{n} a_{n k}^{2} \operatorname{Var}\left[X_{k}\right] \\
& =\sum_{k=1}^{n}\left(\frac{b_{n k}}{s_{n}}\right)^{2} \operatorname{Var}\left[X_{k}\right] \\
& =\frac{\sum_{k=1}^{n} b_{n k}^{2} \operatorname{Var}\left[X_{k}\right]}{s_{n}^{2}} \\
& =\frac{\sum_{k=1}^{n} b_{n k}^{2} \operatorname{Var}\left[X_{k}\right]}{\sum_{k=1}^{n} b_{n k}^{2} \operatorname{Var}\left[X_{k}\right]} \\
& =1,
\end{aligned}
$$

where the last step is allowed because $\operatorname{Var}\left[X_{k}\right] \geq \beta(1-\beta)$ for all $1 \leq k \leq n, 0 \leq b_{n k} \leq C(n)$ for all $1 \leq k \leq n$, and $\sum_{k=1}^{n} b_{n k}=n$, which means that $\sum_{k=1}^{n} b_{n k}^{2} \operatorname{Var}\left[X_{k}\right]>0$. 
Because our construction of $\left\{X_{k}\right\}$ and $\left\{a_{n k}: 1 \leq k \leq n\right\}$ satisfies the preconditions for Lemma 2, applying Lemma 2 yields

$$
\sum_{k=1}^{n} a_{n k} X_{k} \rightarrow N(0,1) \text { as } n \rightarrow \infty .
$$

Now, by straightforward scaling and shifting arguments, we see that

$$
\begin{aligned}
& \sum_{k=1}^{n} a_{n k} X_{k} \rightarrow N(0,1) \text { as } n \rightarrow \infty \\
& \Longleftrightarrow \frac{1}{s_{n}} \sum_{k=1}^{n} b_{n k}\left(Y_{k}-\mathbb{E}\left[Y_{k}\right]\right) \rightarrow N(0,1) \text { as } n \rightarrow \infty \\
& \Longleftrightarrow \sum_{k=1}^{n}\left(b_{n k} Y_{k}-b_{n k} \mathbb{E}\left[Y_{k}\right]\right) \rightarrow N\left(0, s_{n}^{2}\right) \text { as } n \rightarrow \infty \\
& \Longleftrightarrow \sum_{k=1}^{n} b_{n k} Y_{k}-\sum_{k=1}^{n} b_{n k} \mathbb{E}\left[Y_{k}\right] \rightarrow N\left(0, s_{n}^{2}\right) \text { as } n \rightarrow \infty \\
& \Longleftrightarrow \sum_{k=1}^{n} b_{n k} Y_{k} \rightarrow N\left(\sum_{k=1}^{n} b_{n k} \mathbb{E}\left[Y_{k}\right], \sum_{k=1}^{n} b_{n k}^{2} \operatorname{Var}\left[Y_{k}\right]\right) \text { as } n \rightarrow \infty
\end{aligned}
$$

as desired.

We also require the following simple lemma.

Lemma 4. Given a normally distributed variable $X \sim \mathcal{N}(\mathbb{E}[X], \operatorname{Var}[X])$ with $\mathbb{E}[X] \in$ $\left[\mu_{\text {min }}, \mu_{\text {max }}\right]$ and $\operatorname{Var}[X] \in\left[\sigma_{\text {min }}^{2}, \sigma_{\text {max }}^{2}\right]$, the following is true.

Case 1: if $\mu_{\max }>k$ :

$$
\begin{aligned}
& \operatorname{Pr}[X>k] \leq \operatorname{Pr}\left[Y \sim \mathcal{N}\left(\mu_{\text {max }}, \sigma_{\text {min }}^{2}\right)>k\right] \\
& \operatorname{Pr}[X>k] \geq \operatorname{Pr}\left[Y \sim \mathcal{N}\left(\mu_{\text {min }}, \sigma_{\text {max }}^{2}\right)>k\right]
\end{aligned}
$$

Case 2: if $\mu_{\max }<k$ :

$$
\begin{aligned}
& \operatorname{Pr}[X>k] \leq \operatorname{Pr}\left[Y \sim \mathcal{N}\left(\mu_{\text {max }}, \sigma_{\text {max }}^{2}\right)>k\right] \\
& \operatorname{Pr}[X>k] \geq \operatorname{Pr}\left[Y \sim \mathcal{N}\left(\mu_{\text {min }}, \sigma_{\text {min }}^{2}\right)>k\right]
\end{aligned}
$$

Proof. For both upper bounds, we want to minimize the value of $\Phi\left(\frac{k-\mathbb{E}[X]}{\operatorname{Var}[X]}\right)$, where $\Phi$ is the CDF of the standard normal distribution. Because $\Phi$ is monotonically increasing, this is equivalent to minimizing the value of $\frac{k-\mathbb{E}[X]}{\operatorname{Var}[X]}$. It is clear that $k-\mu_{\max }<k-\mu_{\text {min }}$. Now, if $k-\mu_{\max }<0$, then

$$
\frac{k-\mu_{\max }}{\sigma_{\min }}<\frac{k-\mu_{\max }}{\sigma_{\max }}
$$


However, if $k-\mu_{\max }>0$, then

$$
\frac{k-\mu_{\max }}{\sigma_{\max }}<\frac{k-\mu_{\max }}{\sigma_{\min }}
$$

For both lower bounds, we want to maximize the value of $\Phi\left(\frac{k-\mathbb{E}[X]}{\operatorname{Var}[X]}\right)$. Because $\Phi$ is monotonically increasing, this is equivalent to maximizing the value of $\frac{k-\mathbb{E}[X]}{\operatorname{Var}[X]}$. As in the above case, it is clear that $k-\mu_{\min }>k-\mu_{\max }$. Now, if $k-\mu_{\min }<0$, then

$$
\frac{k-\mu_{\min }}{\sigma_{\max }}>\frac{k-\mu_{\min }}{\sigma_{\min }}
$$

However, if $k-\mu_{\min }>0$, then

$$
\frac{k-\mu_{\min }}{\sigma_{\min }}>\frac{k-\mu_{\min }}{\sigma_{\max }}
$$

Finally, by definition, $\operatorname{Erf}(\infty)=1$ and $\operatorname{Erf}(-\infty)=-1$, where $\operatorname{Erf}(\cdot)$ denotes the (Gauss) error function,

$$
\operatorname{Erf}(z)=\frac{2}{\sqrt{\pi}} \int_{0}^{z} e^{-t^{2}} d t
$$

We will use this fact repeatedly throughout the proof of the theorem, which we now turn to.

Proof of Theorem 2. Given a total number of voters $n$, let us define two random variables, $X_{n}^{D}$ and $X_{n}^{M}$, where $X_{n}^{D}$ denotes the number of correct votes under the direct voting mechanism $D$, and $X_{n}^{M}$ represents the (weighted) number of correct votes under GREEDYCAP. We are interested in comparing $P_{n}^{D}=\operatorname{Pr}\left[X_{n}^{D}>n / 2\right]$ and $P_{n}^{M}=\operatorname{Pr}\left[X_{n}^{M}>n / 2\right]$.

Let $V=\left\{V_{1}, \ldots, V_{n}, \ldots\right\}$ be a sequence of independent Bernoulli random variables in which $V_{i}$ represents the vote of voter $i$; i.e., each $V_{i}$ has success probability $p_{i} \in[\beta, 1-\beta]$ for $\beta \in(0,1 / 2)$. Using $V$, we define a sequence of instances indexed by $n$, where each instance consists of the first $n$ voters in $V$. Let $\left\{b_{n i}^{D}: 1 \leq i \leq n\right\}$ and $\left\{b_{n i}^{M}: 1 \leq i \leq n\right\}$ be triangular arrays of nonnegative integers that denote the weight of each voter under direct voting and GREEDYCAP, respectively. Under direct voting, $b_{n i}^{D}=1$ for all $1 \leq i \leq n$. Note that, in this case, $0 \leq b_{n i}^{D} \leq C(n)$ for all voters $i$, where $C(n)$ is the maximum amount of weight any voter can accumulate, and $\sum_{i=1}^{n} b_{n i}^{D}=n$. Now, in the delegative case, let $b_{n i}^{M}=w_{n i}$ for all $1 \leq i \leq n$, where $w_{n i} \in \mathbb{Z}_{\geq 0}$ is the total weight accumulated by voter $i$ in instance $n$ (note that voters who choose to delegate have weight zero). Because each voter cannot accumulate weight greater than $C(n)$, we have that $0 \leq w_{n i} \leq C(n)$ for all voters $i$, and $\sum_{i=1}^{n} w_{n i}=n$.

Note that, given a population of voters of size $n, X_{n}^{D}=\sum_{i=1}^{n} b_{n i}^{D} V_{i}=\sum_{i=1}^{n} V_{i}$ and $X_{n}^{M}=\sum_{i=1}^{n} b_{n i}^{M} V_{i}=\sum_{i=1}^{n} w_{n i} V_{i}$. Now, because $X_{n}^{D}$ and $X_{n}^{M}$ both satisfy the conditions 
under which Lemma 3 holds, we may apply Lemma 3 to establish that $X_{n}^{D}$ and $X_{n}^{M}$ are approximately normally distributed as $n$ goes to infinity; i.e.,

$$
X_{n}^{D}=\sum_{i=1}^{n} V_{i} \rightarrow N\left(\sum_{i=1}^{n} \mathbb{E}\left[V_{i}\right], \sum_{i=1}^{n} \operatorname{Var}\left[V_{i}\right]\right) \text { as } n \rightarrow \infty
$$

and

$$
X_{n}^{M}=\sum_{i=1}^{n} w_{n i} V_{i} \rightarrow N\left(\sum_{i=1}^{n} w_{n i} \mathbb{E}\left[V_{i}\right], \sum_{i=1}^{n} w_{n i}^{2} \operatorname{Var}\left[V_{i}\right]\right) \text { as } n \rightarrow \infty .
$$

Therefore, we can use the following formulas, which are well-known from normal distributions.

$$
\begin{aligned}
& P_{n}^{D} \approx \int_{n / 2}^{n} \frac{1}{\sqrt{2 \pi \operatorname{Var}\left[X_{n}^{D}\right]}} \exp \left(\frac{-\left(x-\mathbb{E}\left[X_{n}^{D}\right]\right)^{2}}{2 \operatorname{Var}\left[X_{n}^{D}\right]}\right) d x \\
& P_{n}^{M} \approx \int_{n / 2}^{n} \frac{1}{\sqrt{2 \pi \operatorname{Var}\left[X_{n}^{M}\right]}} \exp \left(\frac{-\left(x-\mathbb{E}\left[X_{n}^{M}\right]\right)^{2}}{2 \operatorname{Var}\left[X_{n}^{M}\right]}\right) d x
\end{aligned}
$$

Indeed, throughout this proof, we will assume that $P_{n}^{D}$ and $P_{n}^{M}$ are exactly equal to these quantities; this is because Lemma 3 says that as $n$ goes to infinity, this approximation becomes arbitrarily accurate.

Note that, from above, the PG property means that there exists $\varepsilon$ such that $P_{n}^{M}-P_{n}^{D}>\varepsilon$ for at least one graph $G_{n}$ on $n$ vertices for all suitably large $n$. Similarly, the DNH property corresponds to $P_{n}^{D}-P_{n}^{M}<\varepsilon$ for all graphs $G_{n}$ on $n$ vertices for suitably large $n$ and all values of $\varepsilon$. We show that these two properties hold.

For the PG property, we construct a simple family of examples where the property is satisfied. Let the social graph $G$ be composed of pairs of nodes with one competent voter and one incompetent voter with an edge pointing to the competent voter. The competent voters have competence $1-\beta$ and the incompetent voters have competence $\beta$. If the voters vote independently, the symmetry between the competent and incompetent voters makes it clear that $P_{n}^{D}=1 / 2$. Under Algorithm 1, the incompetent voters all delegate to the competent voters. We now have $\frac{n}{2}$ independent voters who each have one vote of weight two and competence $1-\beta$. By the Condorcet Jury Theorem (Grofman et al., 1983), it follows that $P_{n}^{M}$ approaches 1 .

In the remainder of the proof, therefore, we focus on establishing the DNH property. We first show that

$$
\operatorname{Var}\left[X_{n}^{D}\right] \in[\beta(1-\beta) n, n / 4] .
$$

Indeed, $X_{n}^{D}=\sum_{i=1}^{n} V_{i}$, where $V_{i}$ is the Bernoulli random variable representing the vote of voter $i$. In particular, $V_{i} \sim \operatorname{Bernoulli}\left(p_{i}\right)$, where $p_{i} \in[\beta, 1-\beta]$ is the competence level of voter $i$. Because all voters vote independently, $\operatorname{Var}\left[X_{n}^{D}\right]=\sum_{i=1}^{n} \operatorname{Var}\left[V_{i}\right]$, and

$$
\operatorname{Var}\left[V_{i}\right]=p_{i}\left(1-p_{i}\right) \in\left[\beta(1-\beta),(1 / 2)^{2}\right] .
$$


This establishes Equation (27).

Now, let us separate the instances into two cases:

1. $\left|\mathbb{E}\left[X_{n}^{D}\right]-\frac{n}{2}\right|>\frac{n}{\log n}$.

2. $\left|\mathbb{E}\left[X_{n}^{D}\right]-\frac{n}{2}\right| \leq \frac{n}{\log n}$.

Case 1. In this case, we can give strong lower bounds on both $P_{n}^{D}$ and $P_{n}^{M}$.

Subcase 1: $\mathbb{E}\left[X_{n}^{D}\right]<n / 2-n / \log n$. By Equation (27), $\operatorname{Var}\left[X_{n}^{D}\right] \leq n / 4<n$. Because $\mathbb{E}\left[X_{n}^{D}\right]<n / 2$, by Lemma 4 we have

$$
P_{n}^{D}<\int_{\frac{n}{2}}^{n} \frac{1}{\sqrt{2 \pi n}} e^{\frac{-\left(x-\frac{n}{2}+\frac{n}{\log n}\right)^{2}}{2 n}} d x .
$$

This is equivalent to

$$
P_{n}^{D}<\frac{1}{2}\left(\operatorname{Erf}\left(\frac{\sqrt{n}(2+\log n)}{2 \sqrt{2} \log n}\right)-\operatorname{Erf}\left(\frac{\sqrt{n}}{\sqrt{2} \log n}\right)\right)
$$

As $n$ approaches infinity, both arguments go to infinity, and therefore (as $\operatorname{Erf}(\infty)=1) P_{n}^{D}$ approaches 0 . This means that, no matter the value of $P_{n}^{M}$, DNH is satisfied.

Subcase 2: $\mathbb{E}\left[X_{n}^{D}\right]>n / 2+n / \log n$. We now examine the maximum possible value of $\operatorname{Var}\left[X_{n}^{M}\right]=\sum_{i=1}^{n} w_{n i}^{2} \operatorname{Var}\left[V_{i}\right]$, where $w_{n i}$ is the total weight accumulated by voter $i$ and, again, $V_{i}$ is the Bernoulli random variable representing the vote of voter $i$. Additionally, $\operatorname{Var}\left[V_{i}\right] \in[\beta(1-\beta), 1 / 4]$, and applying this yields

$$
\operatorname{Var}\left[X_{n}^{M}\right] \leq \frac{1}{4} \cdot \sum_{i=1}^{n} w_{n i}^{2}
$$

Because each voter can accumulate at most weight $C(n)$, by the convexity of $x^{2}$, we can see that this is maximized when the maximum number of voters have weight exactly $C(n)$. Therefore, we have

$$
\operatorname{Var}\left[X_{n}^{M}\right] \leq \frac{1}{4} \cdot \sum_{i=1}^{\lceil n / C(n)\rceil} C(n)^{2}<n C(n) .
$$

Because $\mathbb{E}\left[X_{n}^{D}\right]>n / 2$, by Lemma 4 we have

$$
P_{n}^{M}>\int_{\frac{n}{2}}^{n} \frac{1}{\sqrt{2 \pi n C(n)}} e^{\frac{-\left(x-\frac{n}{2}+\frac{n}{\log n}\right)^{2}}{2 n C(n)}} d x
$$


This simplifies to

$$
P_{n}^{M}>\frac{1}{2}\left(\operatorname{Erf}\left(\frac{\sqrt{n}(\log n-2)}{2 \sqrt{2 C(n)} \log n}\right)+\operatorname{Erf}\left(\frac{\sqrt{n}}{\sqrt{2 C(n)} \log n}\right)\right) .
$$

As $n$ approaches infinity, both arguments go to infinity, and $P_{n}^{M}$ approaches 1 . Therefore, no matter what the value of $P_{n}^{D}$, DNH is satisfied.

Case 2. In this case, we split the argument into two further subcases:

1. The number of voters who delegate is greater than $n / g(n)$, where $g(n)$ is $o(\log n)$ and $\omega\left(C(n)^{2}\right)$.

2. The number of voters who delegate is less than or equal to $n / g(n)$.

Subcase 1: Due to delegation, we have $\mathbb{E}\left[X_{n}^{M}\right]-\mathbb{E}\left[X_{n}^{D}\right] \geq n \alpha / g(n)$. We can now bound the mean by

$$
\mathbb{E}\left[X_{n}^{M}\right] \geq \frac{n}{2}-\frac{n}{\log n}+\frac{n \alpha}{g(n)} .
$$

Therefore, because $g(n)=o(\log n), \mathbb{E}\left[X_{n}^{M}\right]>n / 2$ as $n$ increases. As before, we also know that $\operatorname{Var}\left[X_{n}^{M}\right]$ is bounded from above by $n C(n)$, and therefore, by Lemma 4 ,

$$
P_{n}^{M} \geq \int_{\frac{n}{2}}^{n} \frac{1}{\sqrt{2 \pi n C(n)}} e^{\frac{-\left(x-\frac{n}{2}+\frac{n}{\log n}-\frac{n \alpha}{g(n)}\right)^{2}}{2 n C(n)}} d x
$$

We would like to show that this integral goes to 1 as $n$ goes to infinity.

This is equivalent to

$$
\frac{1}{2}\left(\operatorname{Erf}\left(\frac{\frac{n}{2}-\frac{n \alpha}{g(n)}+\frac{n}{\log n}}{\sqrt{2 n C(n)}}\right)-\operatorname{Erf}\left(\frac{\sqrt{n}\left(-\frac{\alpha}{g(n)}+\frac{1}{\log n}\right)}{\sqrt{2 C(n)}}\right)\right) .
$$

Note that as $n$ goes to infinity, the first argument goes to infinity and the second argument goes to negative infinity when $g(n)=o(\log n)$. Therefore, $P_{n}^{M}$ goes to 1 , satisfying DNH.

Subcase 2: In this case, most voters remain independent. We will argue that although the delegation does impact the variance, this impact will get arbitrarily small as $n$ grows larger, implying that the loss will get arbitrarily small.

Let us index the voters according to what happens in the delegation scheme. Let the first $n_{1}$ indexed voters represent those who remain independent and do not get delegated a vote. Let the next $n_{2}$ indexed voters be those who got delegated at least one vote. Finally, the last $n-n_{1}-n_{2}$ indexed voters are those who delegated their vote to another voter. Based on our assumption above, we know that $\lim _{n \rightarrow \infty} \frac{n_{1}}{n}=1$; most voters remain independent and unaffected by the delegation scheme. 
Additionally, note that the mean will be slightly different in the two schemes, but this is to our advantage because the mean will improve in the delegation scheme due to "uphill" delegation.

Therefore, given

$$
P_{n}^{D}=\int_{\frac{n}{2}}^{n} \frac{1}{\sqrt{2 \pi \operatorname{Var}\left[X_{n}^{D}\right]}} e^{\frac{-\left(x-\mathbb{E}\left[X_{n}^{D}\right]\right)^{2}}{2 \operatorname{Var}\left[X_{n}^{D}\right]}} d x
$$

and

$$
P_{n}^{M}=\int_{\frac{n}{2}}^{n} \frac{1}{\sqrt{2 \pi \operatorname{Var}\left[X_{n}^{M}\right]}} e^{\frac{-\left(x-\mathbb{E}\left[X_{n}^{M}\right]\right)^{2}}{2 \operatorname{Var}\left[X_{n}^{M}\right]}} d x
$$

because $\mathbb{E}\left[X_{n}^{M}\right] \geq \mathbb{E}\left[X_{n}^{D}\right]$, we can say that

$$
P_{n}^{M} \geq \int_{\frac{n}{2}}^{n} \frac{1}{\sqrt{2 \pi \operatorname{Var}\left[X_{n}^{M}\right]}} e^{\frac{-\left(x-\mathbb{E}\left[X_{n}^{D}\right]\right)^{2}}{2 \operatorname{Var}\left[X_{n}^{M}\right]}} d x .
$$

Now, we have to relate $\operatorname{Var}\left[X_{n}^{M}\right]$ and $\operatorname{Var}\left[X_{n}^{D}\right]$. Ideally, we want to show that they are multiplicatively close to each other.

We can decompose the variance of $X_{n}^{D}$.

$$
\operatorname{Var}\left[X_{n}^{D}\right]=\sum_{i=1}^{n_{1}} p_{i}\left(1-p_{i}\right)+\sum_{i=n_{1}+1}^{n} p_{i}\left(1-p_{i}\right) .
$$

Likewise, we can decompose the variance of $X_{n}^{M}$.

$$
\operatorname{Var}\left[X_{n}^{M}\right]=\sum_{i=1}^{n_{1}} p_{i}\left(1-p_{i}\right)+\sum_{i=n_{1}+1}^{n_{1}+n_{2}} w_{n i}^{2} p_{i}\left(1-p_{i}\right)+\sum_{i=n_{1}+n_{2}+1}^{n} 0 .
$$

Therefore, we have

$$
\begin{aligned}
\operatorname{Var}\left[X_{n}^{M}\right]-\operatorname{Var}\left[X_{n}^{D}\right] & =\sum_{i=n_{1}+1}^{n_{1}+n_{2}}\left(w_{n i}^{2}-1\right) p_{i}\left(1-p_{i}\right) \\
& \quad-\sum_{i=n_{1}+n_{2}+1}^{n} p_{i}\left(1-p_{i}\right) \\
& \leq \sum_{i=n_{1}+1}^{n_{1}+n_{2}}\left(w_{n i}^{2}-1\right) p_{i}\left(1-p_{i}\right) \\
\leq & \frac{n_{2}}{4}\left(\max w_{n i}{ }^{2}-1\right)
\end{aligned}
$$




$$
\leq \frac{1}{4} \cdot \frac{n}{g(n)}\left(C(n)^{2}-1\right),
$$

where the last inequality holds because $w_{n i} \leq C(n)$, and $n_{2}$, the number of voters who are delegated to, is at most the number of voters who delegate, which is at most $n / g(n)$ by assumption.

This means that

$$
\operatorname{Var}\left[X_{n}^{M}\right] \leq \operatorname{Var}\left[X_{n}^{D}\right]+\frac{1}{4} \cdot \frac{n}{g(n)}\left(C(n)^{2}-1\right)
$$

and therefore

$$
\begin{aligned}
\frac{\operatorname{Var}\left[X_{n}^{M}\right]}{\operatorname{Var}\left[X_{n}^{D}\right]} & \leq \frac{\operatorname{Var}\left[X_{n}^{D}\right]+\frac{1}{4} \cdot \frac{n}{g(n)}\left(C(n)^{2}-1\right)}{\operatorname{Var}\left[X_{n}^{D}\right]} \\
& =1+\frac{\frac{n}{g(n)}\left(C(n)^{2}-1\right)}{4 \operatorname{Var}\left[X_{n}^{D}\right]}
\end{aligned}
$$

Now, note that by Equation (27),

$$
\operatorname{Var}\left[X_{n}^{D}\right] \geq n \beta(1-\beta)
$$

and therefore

$$
\begin{aligned}
\operatorname{Var}\left[X_{n}^{M}\right] & \leq \operatorname{Var}\left[X_{n}^{D}\right]\left(1+\frac{\frac{n}{g(n)}\left(C(n)^{2}-1\right)}{4 n \beta(1-\beta)}\right) \\
& =\operatorname{Var}\left[X_{n}^{D}\right]\left(1+\frac{1}{g(n)} \cdot \frac{C(n)^{2}-1}{4 \beta(1-\beta)}\right) .
\end{aligned}
$$

Let

$$
\eta=\frac{1}{g(n)} \cdot \frac{C(n)^{2}-1}{4 \beta(1-\beta)}
$$

and note that as $n$ goes to infinity, $\eta$ goes to 0 because we chose $g(n)$ to grow asymptotically more quickly than $C(n)^{2}$.

Therefore, revisiting the original integrals, we have

$$
P_{n}^{D}=\int_{\frac{n}{2}}^{n} \frac{1}{\sqrt{2 \pi \operatorname{Var}\left[X_{n}^{D}\right]}} e^{\frac{-\left(x-\mathbb{E}\left[X_{n}^{D}\right]\right)^{2}}{2 \operatorname{Var}\left[X_{n}^{D}\right]}} d x
$$

and

$$
P_{n}^{M} \geq \int_{\frac{n}{2}}^{n} \frac{1}{\sqrt{2 \pi \operatorname{Var}\left[X_{n}^{D}\right](1+\eta)}} e^{\frac{-\left(x-\mathbb{E}\left[X_{n}^{D}\right]\right)^{2}}{2 \operatorname{Var}\left[X_{n}^{D}\right](1+\eta)}} d x
$$

Simplifying the above yields

$$
P_{n}^{D}=\frac{1}{2}\left(\operatorname{Erf}\left(\frac{n-\mathbb{E}\left[X_{n}^{D}\right]}{\sqrt{2 \operatorname{Var}\left[X_{n}^{D}\right]}}\right)-\operatorname{Erf}\left(\frac{n-2 \mathbb{E}\left[X_{n}^{D}\right]}{2 \sqrt{2 \operatorname{Var}\left[X_{n}^{D}\right]}}\right)\right)
$$


and

$$
\begin{aligned}
P_{n}^{M} \geq \frac{1}{2} & \left(\operatorname{Erf}\left(\frac{n-\mathbb{E}\left[X_{n}^{D}\right]}{\sqrt{2 \operatorname{Var}\left[X_{n}^{D}\right](1+\eta)}}\right)\right. \\
& \left.-\operatorname{Erf}\left(\frac{n-2 \mathbb{E}\left[X_{n}^{D}\right]}{2 \sqrt{2 \operatorname{Var}\left[X_{n}^{D}\right](1+\eta)}}\right)\right)
\end{aligned}
$$

Furthermore, again by Equation (27), we know that $\operatorname{Var}\left[X_{n}^{D}\right] \in[\beta(1-\beta) n, n / 4]$ and therefore $\sqrt{\operatorname{Var}\left[X_{n}^{D}\right]}=\sqrt{c n}$, where $c \in[\beta(1-\beta), 1 / 4]$. From this, note that as $n$ goes to infinity, the argument to the first error function in each expression goes to infinity.

Let

$$
h_{1}(n)=\frac{n-2 \mathbb{E}\left[X_{n}^{D}\right]}{2 \sqrt{2 \operatorname{Var}\left[X_{n}^{D}\right]}}
$$

be the argument to the second error function in (31), and let

$$
h_{2}(n)=\frac{n-2 \mathbb{E}\left[X_{n}^{D}\right]}{2 \sqrt{2 \operatorname{Var}\left[X_{n}^{D}\right](1+\eta)}}
$$

be the argument to the second error function in (32). As $n$ goes to infinity, note that the argument in (33) must go to one of four states: infinity, negative infinity, zero, or a constant. In the case that it goes to infinity, negative infinity, or zero, the presence of the extra $\frac{1}{\sqrt{1+\eta}}$ term in (34) does nothing to change the sign of the arguments, and therefore they each converge to the same state (infinity, negative infinity, or zero) as $n$ approaches infinity. When the argument in (33) goes to a constant, note that as $n$ goes to infinity, $\eta$ goes to 0 , and therefore the two converge once again.

We conclude that (an upper bound on) the difference between $P_{n}^{D}$ and $P_{n}^{M}$ converges to 0 , and hence DNH is satisfied.

\section{Discussion}

We wrap up with a discussion of two central issues.

How realistic is the model? We revisit an important point, which has already come up several times, including in Section 1. Our assumption that voters only delegate their votes to more competent voters is clearly restrictive. But we feel it allows us, in a sense, to distill the essence of liquid democracy (e.g., by avoiding complications that have to do with delegation cycles) and focus on central issues such as vote correlation. Moreover, as noted earlier, our negative result - Theorem 1 - is especially powerful in this model, that is, it holds despite the foregoing assumption. And the positive result - Theorem 2 - should (informally speaking) still hold in a relaxed model where voters may delegate their votes to less competent voters, as long as the average competence level increases by a constant due to delegation. We view this as a realistic assumption. 
Beyond binary issues. In our model, there are only two alternatives, one correct and one incorrect. While this setting is of practical importance, it is natural to ask whether our results extend to the case of three or more alternatives. However, there are several obstacles.

First, a representation of the ground truth, and of voters' perceptions thereof, is required. A popular option is the Mallows Model (Mallows, 1957), where the ground truth is a ranking of the alternatives, and the probability that a voter cast a given ranking as her vote decreases exponentially with its "distance" from the ground truth, in a way that depends on a (competence) parameter $\phi_{i}$. This model coincides with ours (using a suitable transformation between $\phi_{i}$ and $p_{i}$ ) when the number of alternatives is 2 .

Second, we have assumed that votes are aggregated using the majority rule, which is the only reasonable voting rule when there are two alternatives. By contrast, when choosing among three or more alternatives, there are many voting rules one can use.

To conclude, any attempt to extend our model and results beyond the case of two alternatives would have to address not only technical challenges, but also conceptual ones.

\section{Acknowledgments}

A preliminary version of this paper (Kahng et al., 2018) appeared in the proceedings of AAAI'18. This work was partially supported by NSF grants IIS-1350598, IIS-1714140, CCF1525932, and CCF-1733556; by ONR grants N00014-16-1-3075 and N00014-17-1-2428; and by a Sloan Research Fellowship.

\section{References}

Abramowitz, B., \& Mattei, N. (2018). Flexible representative democracy: An introduction with binary issues. arXiv:1811.02921.

Alger, D. (2006). Voting by proxy. Public Choice, 126(1-2), 1-26.

Bloembergen, D., Grossi, D., \& Lackner, M. (2019). On rational delegations in liquid democracy. In Proceedings of the 33rd AAAI Conference on Artificial Intelligence (AAAI), pp. 1796-1803.

Brandt, F., Conitzer, V., Endriss, U., Lang, J., \& Procaccia, A. D. (Eds.). (2016). Handbook of Computational Social Choice. Cambridge University Press.

Brill, M., \& Talmon, N. (2018). Pairwise liquid democracy. In Proceedings of the 27th International Joint Conference on Artificial Intelligence (IJCAI), pp. 137-143.

Caragiannis, I., \& Micha, E. (2019). A contribution to the critique of liquid democracy. In Proceedings of the 28th International Joint Conference on Artificial Intelligence (IJCAI), pp. 116-122. 
Christoff, Z., \& Grossi, D. (2017). Binary voting with delegable proxy: An analysis of liquid democracy. In Proceedings of the 16th Conference on Theoretical Aspects of Rationality and Knowledge (TARK), pp. 134-150.

Cohensius, G., Mannor, S., Meir, R., Meirom, E., \& Orda, A. (2017). Proxy voting for better outcomes. In Proceedings of the 16th International Conference on Autonomous Agents and Multi-Agent Systems (AAMAS), pp. 858-866.

Gölz, P., Kahng, A., Mackenzie, S., \& Procaccia, A. (2018). The fluid dynamics of liquid democracy. In Proceedings of the 13th Conference on Web and Internet Economics (WINE), pp. 188-202.

Green-Armytage, J. (2015). Direct voting and proxy voting. Constitutional Political Economy, 26(2), 190-220.

Grofman, B., Owen, G., \& Feld, S. L. (1983). Thirteen theorems in search of the truth. Theory and Decision, 15(3), 261-278.

Hoeffding, W. (1963). Probability inequalities for sums of bounded random variables. Journal of the American Statistical Association, 58(301), 13-30.

Kahng, A., Mackenzie, S., \& Procaccia, A. (2018). Liquid democracy: An algorithmic perspective. In Proceedings of the 32nd AAAI Conference on Artificial Intelligence (AAAI), pp. 1095-1102.

Kling, C. C., Kunegis, J., Hartmann, H., Strohmaier, M., \& Staab, S. (2015). Voting behaviour and power in online democracy. In Proceedings of the 9th International AAAI Conference on Web and Social Media (ICWSM), pp. 208-217.

Mallows, C. L. (1957). Non-null ranking models. Biometrika, 44, 114-130.

Miller, J. C. (1969). A program for direct and proxy voting in the legislative process. Public Choice, 7(1), 107-113.

Tullock, G. (1992). Computerizing politics. Mathematical and Computer Modelling, 16(89), 59-65. 